\title{
Recurrent Rectosigmoid Carcinoma
}

National Cancer Institute

\section{Source}

National Cancer Institute. Recurrent Rectosigmoid Carcinoma. NCI Thesaurus. Code C8634.

The reemergence of rectosigmoid carcinoma after a period of remission. 